\title{
Flow-driven transition and associated velocity profiles in a nematic liquid-crystal cell
}

\author{
S. A. Jewell, ${ }^{1}$ S. L. Cornford, ${ }^{2}$ F. Yang, ${ }^{1}$ P. S. Cann, ${ }^{1}$ and J. R. Sambles ${ }^{1}$ \\ ${ }^{1}$ Electromagnetic Materials Group, School of Physics, University of Exeter, Stocker Road, Exeter EX4 4QL, United Kingdom \\ ${ }^{2}$ Hewlett-Packard Laboratories, Filton Road, Stoke Gifford, Bristol BS34 8QZ, United Kingdom
}

(Received 16 March 2009; revised manuscript received 14 September 2009; published 26 October 2009)

\begin{abstract}
The alignment properties and distribution of flow speed during Poiseuille flow through a microchannel of a nematic liquid crystal in a cell with homeotropic surface alignment has been measured using a combination of conoscopy, fluorescence confocal polarizing microscopy, and time-lapse imaging. Two topologically distinct director profiles, with associated fluid velocity fields, are found to exist with the preferred state dictated by the volumetric flow rate of the liquid crystal. The results show excellent agreement with model data produced using the Ericksen-Leslie nematodynamics theory.
\end{abstract}

DOI: 10.1103/PhysRevE.80.041706

PACS number(s): 42.70.Df, 61.30.Gd, 47.85.Np

\section{INTRODUCTION}

The cross-disciplinary area of microfluidics, the study of the flow of liquids through narrow channels, has applications ranging from small-scale "lab-on-a-chip" biosensors to industrial injection molding processes. The confinement of a fluid between walls separated on the micron scale can impose significant conditions on the flow behavior of the material. For complex fluids, such as liquid crystals, the added constraint of molecular alignment on the flowing material can lead to phase changes in lyotropic liquid crystals and the formation of defects when extruding polymeric liquid crystals. Thus understanding the Poiseuille flow (fluid flow through a channel due to a pressure difference) of liquid crystals through micron-scale channels is of increasing significance. In this study we explore in detail the flow of a nematic thermotropic liquid crystal through a $35 \mu \mathrm{m}$ deep channel under pressure-driven flow.

The fluid dynamics of liquid crystals is complex due to the inherent anisotropy. Several approaches have previously been used to probe the viscoelastic properties of nematic liquid crystals. It is well known that flow is induced during the reorientation of the liquid-crystal director field in response to the application of an electric field to a liquidcrystal layer, and this has important consequences on the response time of liquid crystal displays [1,2]. Colloidal dispersions of electrically neutral nanoparticles in liquid crystals have previously enabled electrically induced backflow close to the walls of a nematic liquid-crystal cell [3] and the flow parallel to smectic layers in ferroelectric liquid crystals [4] to be visualized. Numerical modeling of the Poiseuille flow of liquid-crystal polymers in channels of varying width [5] has shown that modifications to the channel dimensions has a significant impact on the order parameter of the flowing liquid crystal. Other studies have explored how pressuredriven flow alignment is influenced by the presence of fixed particles and the interaction between flow and topological defects [6]. Experimentally determining the fluid velocity at discrete points through the depth of a nematic liquid-crystal cell has until now been a challenge due to the requirement of accurately probing the fluid velocity at well-defined depths within the channel. One approach used to examine the flow of isotropic fluids close to channel walls was to disperse fluorescent nanoparticles in water undergoing an oscillatory shear flow using a charge-coupled device (CCD) camera to collect images of the particle motion using direct illumination for particles in the bulk of the cell and total internal reflection fluorescence for particles close to the walls [7].

In this paper, we present a thorough experimental and model treatment of the pressure-driven flow of a homeotropically aligned nematic liquid crystal though a microchannel. The use of a conoscopic technique allows the reorientation of the bulk of the liquid crystal during the flow process to be observed in real time. The use of a fluorescent confocal polarizing microscopy technique then allows a more detailed exploration of the director profile as a function of depth through the channel. Finally, the use of microparticles in a time-lapse imaging procedure allows the actual velocity distribution across the microchannel to be probed.

\section{THEORY}

While the liquid crystal's steady-state director configuration in a microchannel is strictly a three-dimensional problem, much of its behavior can be understood in terms of a one-dimensional model. Two quantities must be calculated, the fluid velocity $\boldsymbol{u}$ and the director $\boldsymbol{n}$, a unit vector parallel to the average long molecular axis. Far from the inlet and outlet pipes all flow is parallel to the channel (i.e., along the $x$ axis) and assumed to be uniform with respect to $x$ with the director confined to the $x-z$ plane. For a channel of a width that is much greater than its depth the problem can be reduced to a single dimension, varying along the $z$ axis alone.

A simple one-dimensional model of the liquid crystal's steady-state behavior, derived from the Ericksen-Leslie equations [8,9], can be written in terms of the angle of inclination between the director and the $z$ axis, $\theta$, and the $x$ component of the flow velocity, $\boldsymbol{u}$. Fluid inertia may be neglected [10] so that the linear momentum balance equation becomes

$$
\frac{d}{d z}\left(\eta(\theta) \frac{d u}{d z}\right)=G
$$

where $G$ is the pressure gradient along the $x$ axis and $\eta(\theta)$ is an effective viscosity which depends on $\theta$ and three independent combinations of the Leslie viscosities $\alpha_{1}, \ldots, \alpha_{6}$. This is 
TABLE I. Relevant material parameters of $5 \mathrm{CB}$, taken from Ref. [9].

\begin{tabular}{lll}
\hline \hline Parameter & & Value \\
\hline Elastic constants & $k_{11}, k_{33}$ & $6.2,8.2 \mathrm{pN}$ \\
Leslie viscosities & $\alpha_{2}, \alpha_{3}, \alpha_{1}, \alpha_{4}+\alpha_{5}$ & $-81,-3.6,6.0$, \\
& & $130 \mathrm{mPa} \mathrm{s}$ \\
Dielectric constants & $\varepsilon_{\perp}, \varepsilon_{\|}$ & $7,18.5$ \\
\hline \hline
\end{tabular}

to be solved alongside an equation balancing elastic torques due to spatial variation in $\theta$ with the tendency for shear forces to realign the liquid crystal,

$$
k \frac{d^{2} \theta}{d z^{2}}-\left(\alpha_{3} \sin \theta-\alpha_{2} \cos \theta\right) \frac{d u}{d z}=0,
$$

where $\left(k_{11}\right)$ and $\left(k_{33}\right)$ are the splay and bend elastic coefficients, respectively. Numerical solutions to Eqs. (1) and (2) were found using a finite volume method. We also considered the more complicated case where an electric field is applied between the upper and lower surfaces of the cell. Table I gives values for all the parameters which appear in Eqs. (1) and (2) corresponding to 5CB.

Equations (1) and (2) are solved with nonslip boundary conditions where $\boldsymbol{u}(0)=\boldsymbol{u}(d)=0$. When considering the boundary conditions for the tilt profile two sets of parameters describe the homeotropic anchoring equally well [11]: $\theta(0)$ $=\theta(d)=0$ and $\theta(0)=0$ and $\theta(d)=\pi$. Of these two, the first set leads to a director profile which is vertical at the cell's midplane and walls, while the second set produces a director profile that is horizontal at the midplane. We label these two possible profiles, illustrated in Fig. 1, $V$ (vertical) and $H$ (horizontal) states, respectively. Notice that both of these configurations are mirror symmetric about the cell's midplane: this symmetry will be used to infer the presence of these states in our conoscopic experiments.

In the absence of flow, only the $V$ state is observed. Since the $H$ state involves a continuous distortion across the cells, its elastic free energy

$$
F=\int_{0}^{d}\left(k_{11} \sin ^{2} \theta+k_{33} \cos ^{2} \theta\right)\left(\frac{d \theta}{d z}\right)^{2} d z=0
$$

is greater than that of the $V$ state computed by the same formula. The same is true at low flow rates. However, as Fig. 2 shows, the energy of the $V$ state grows more rapidly with fluid speed, so that the $H$ state eventually becomes energetically favorable. This happens primarily because $\left|\alpha_{2}\right| \gg\left|\alpha_{3}\right|$. In the $V$ state, gradients in $\theta$ grow with gradients in $\boldsymbol{u}$ at a rate approximately proportional to $\left|\alpha_{2}\right|$. The picture is more complicated in the $H$ state, but in the midplane region the curvature grows more slowly at a rate roughly proportional to $\left|\alpha_{3}\right|$.

Why might one expect to observe the state with the lower elastic free energy? After all, it is the torque balance Eq. (2) which governs the director profile, not the minimization of the free energy. Consider a cell in the $V$ state having a nucleation site which perturbs a region bounded horizontally by a
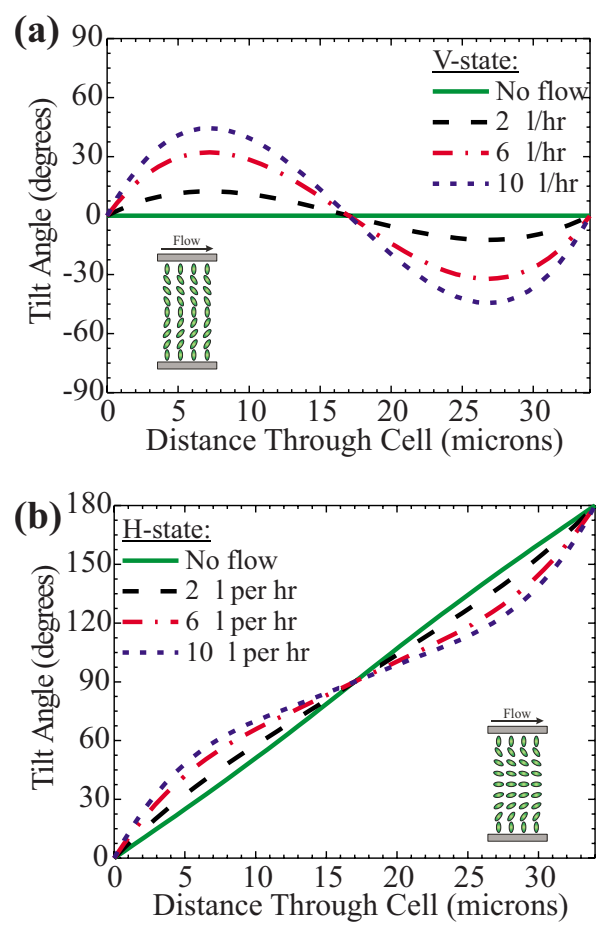

FIG. 1. (Color online) $V$ and $H$ states. Modeled director tilt angle versus depth through a channel $3 \mathrm{~mm}$ wide and $34 \mu \mathrm{m}$ deep for selected volumetric flow rates using viscosity parameters given in Table I for (a) $V$-state and (b) $H$-state flows. The schematic diagrams illustrate the director orientation across the liquid crystal cell.

contour, $R(x, y)$, and extending vertically through the cell so that it evolves toward the $H$ state. Equation (2) is eventually satisfied both inside $R$ and outside, but if the $V$ state has the lower energy, thermodynamic forces will act to shrink it, so that far downstream it will vanish. On the other hand, if the $V$ state has the higher energy, $R$ will grow, as it is swept downstream, eventually filling the channel.

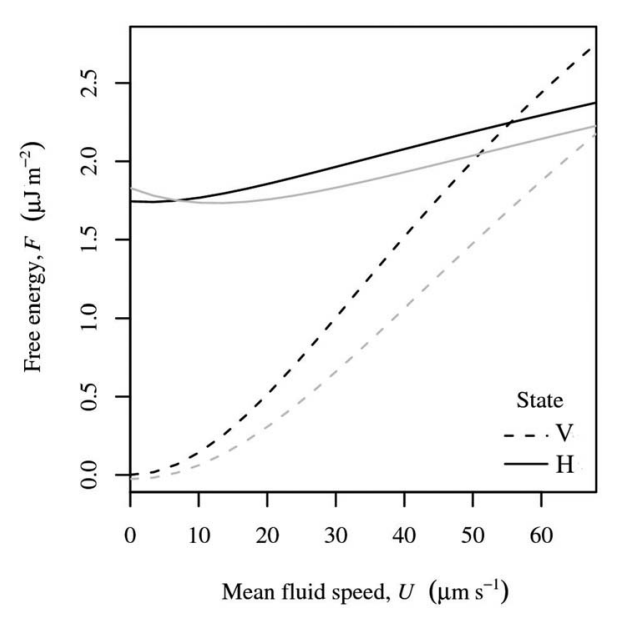

FIG. 2. Calculated free energy of $5 \mathrm{CB}$ per unit area plotted against mean (in $z$ ) fluid speed. The $V$ state has the lower free energy at lower speeds (dark dashes), but the $H$ state (dark solid line) has the lower free energy above a critical mean fluid speed $\mu_{\mathrm{c}}=55 \mu \mathrm{m} \mathrm{s}^{-1}$, where the curves cross. Applying an electric field (lighter curves) increases the critical speed. 


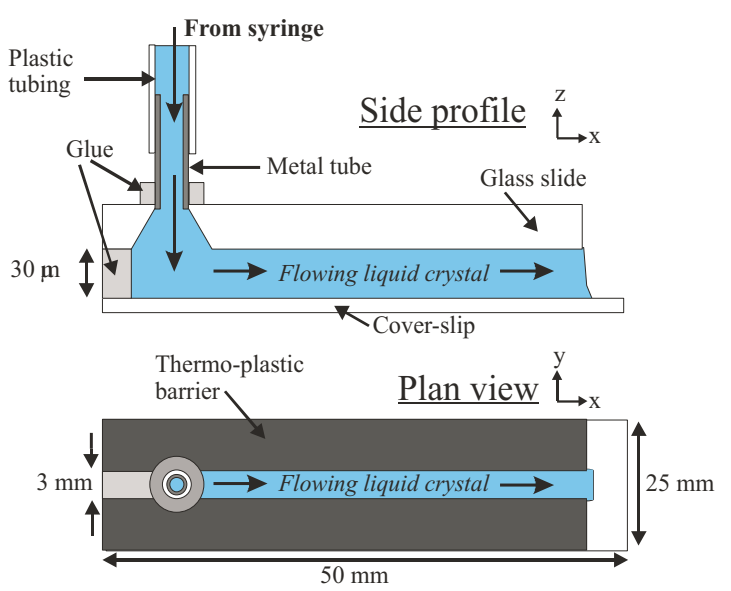

FIG. 3. (Color online) Schematic diagram of the pressure-driven flow cell.

\section{EXPERIMENT AND RESULTS}

Flow channels $30 \mathrm{~mm}$ long, $3 \mathrm{~mm}$ wide, and $\sim 30 \mu \mathrm{m}$ deep were constructed using glass $(n=1.52)$ substrates as the upper and lower layers, as shown in Fig. 3. The superstrate had a $0.8 \mathrm{~mm}$ diameter hole at one end to allow the liquid crystal to enter the cell and this was counter sunk to a diameter of $3 \mathrm{~mm}$ as shown to ensure that the material entered the cell over a large entrance area. The substrates were treated with octadecyltrimethoxysilane to induce homeotropic alignment and the cell was assembled using strips of mylar $\sim 30 \mu \mathrm{m}$ thick and $10 \mathrm{~mm}$ wide to both define the two sides of the channel and act as a spacer. The end of the channel closest to the inlet was sealed using UV-setting glue and the other end was left open to allow the liquid crystal to freely exit the cell. A metal pipe was sealed to the inlet hole and a $100 \mathrm{~mm}$ length of PTFE tubing was used to connect the pipe to a driven syringe containing $5 \mathrm{CB}$ (K15, Merck).

\section{A. Conoscopy measurements}

We observed transitions from the $V$ to the $H$ state, and back, through conoscopic measurements. Conoscopy is a powerful optical tool that allows the optical properties of a slab of birefringent medium to be measured over a range of angles simultaneously. A convergent monochromatic beam passing through a liquid-crystal layer is focused onto the back focal plane of an objective lens to form a real image. Each point in the focal plane receives the light from only one direction and when the sample is viewed between crossed polarizers the wealth of information contained in the resulting interference figures may be used to determine the alignment properties of the liquid-crystal sample. The use of conoscopy in association with a CCD imaging camera allows real-time imaging of the director orientation in a nematic under pressure-driven flow.

For the homeotropically aligned cell explored in this study a transition in the conoscopic image from that predicted for the $V$ state to one for the $H$ state was observed when the syringe pump was increased to a volumetric flow rate of $7 \mu \mathrm{l} / \mathrm{hr}$. To compare these rates with the calculated critical fluid speed $\mu_{\mathrm{c}}=55 \mu \mathrm{m} \mathrm{s}^{-1}$ one can assume that the

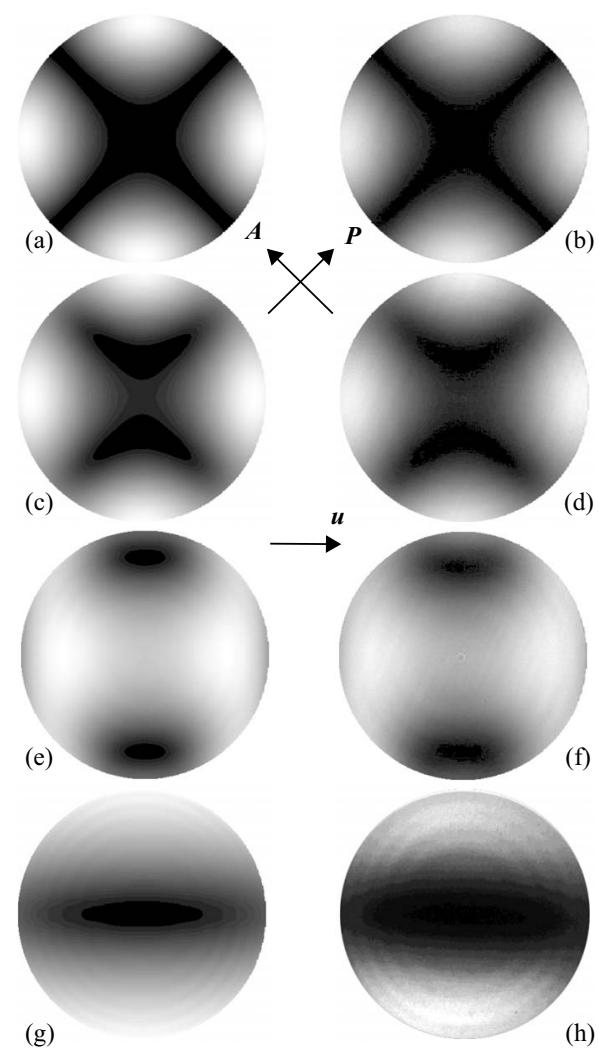

FIG. 4. Theoretical and observed steady-state interference figures. Calculated figures are shown on the left and measurements on the right. The polarizer $(\boldsymbol{P})$ and analyzer $(\boldsymbol{A})$ are crossed and both are inclined at $\pi / 4$ with respect to the direction of flow. When there is no flow, the usual Maltese cross figure ( $a$ and $b$ ) is seen. As the fluid velocity $\boldsymbol{u}$ is increased the cross contracts (c and d) and divides into two dark lobes (e and f). Circular fringes due to thinfilm interference are present but dim in these figures. Above a critical flow rate, a different type of figure is seen, in which circular fringes are clearly visible, and a dark horizontal isochrome is present ( $g$ and $h)$.

flow profile varies quadratically in $y$, and takes on the value $\mu_{\mathrm{c}}$ at the center of the channel. This gives a volumetric flow rate of $7.9 \pm 1 \mu \mathrm{l} / \mathrm{hr}$.

Figure 4 compares interference figures calculated for the ground $(V)$ state, a flowing $V$ state, and a flowing $H$ state, with those observed. In both states, the mirror symmetry of the director profiles leads to figures which are both up-down and left-right symmetric. These are in contrast with the figures which would occur if the director tilted one way, which would only be up-down symmetric. While the cell remains in a $V$ state the isogyres (the dark central cross) contract, then split into two lobes which move apart as the flow speed increases. These distinctive figures have been observed by Waton [12], and can be exploited to estimate the viscosity $\alpha_{2}$. Figures corresponding to the $H$ state are not always so conspicuous, but can include an isochrome, as in Fig. 4, where the phase difference between the ordinary and extraordinary light waves is $2 \pi$. In the case of $5 \mathrm{CB}$, the two states are also distinguished by the amplitude of circular fringes in the conoscopic figures due to interference between waves reflected from the lower and upper surfaces. The ordinary 


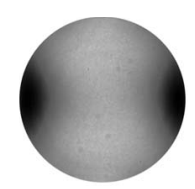

$t=5 \mathrm{~s}$

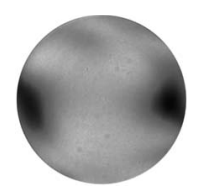

$t=8 \mathrm{~s}$

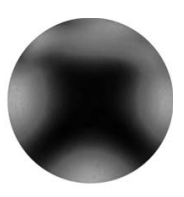

$t=12 \mathrm{~s}$
FIG. 5. A sequence of interference figures taken in the seconds after a $10 \mathrm{~V}$ (peak-to-peak) electric field is applied across the cell. After $5 \mathrm{~s}$, the whole view is still in an $H$ state, then evolves toward a $V$ state nonuniformly. After $12 \mathrm{~s}$, most of the cell is a its homeotropic state.

refractive index of $5 \mathrm{CB}$ is close to that of glass $\left(n_{o}=1.5\right)$, while the extraordinary index is far higher $\left(n_{e}=1.7\right)$, so these fringes are barely visible when the cell is in a $V$ state but quite obvious when it is in an $H$ state.

A cell driven to the $H$ state by shear forces can be forced back into a $V$ state by applying an electric field parallel to the $z$ axis. Figure 2 shows that when a field is applied the free energy of the liquid crystal is reduced more in the $V$ state than it is in the $H$ state. Provided the field is large enough, the $V$ state becomes energetically favorable, and the cell switches to it. This process does not occur across the whole cell simultaneously, but instead spreads across it over several seconds, as can be seen in the sequence of figures shown in Fig. 5. In other words, the transition from $H$ to $V$ (and vice versa) is nucleated.

\section{B. Fluorescent confocal polarizing microscopy measurements}

It is clearer still that the cell adopts two quite different states when the flow channel is imaged under fluorescence confocal polarizing microscopy (FCPM) $[13,14]$. In this experiment, the $5 \mathrm{CB}$ is doped with a low $(\sim 0.01 \%)$ concentration of an anisomeric dye (BTBP, Sigma-Aldrich), which mimics the alignment of the liquid-crystal molecules. When the dye is excited by a polarized laser beam at its excitation wavelength, the fluorescence produced is proportional to $\cos ^{4} \Phi$, where $\Phi$ is the angle between the polarization direction and the dye axis. When used in combination with an optical confocal system the fluorescence at the emission wavelength from a volume $<1 \mu \mathrm{m}^{3}$ can be collected, allowing the director orientation to be determined with a resolution on the submicron scale. To examine the switching of the homeotropic cell both the polarizer and analyzer of the FCPM system were placed parallel to the direction of flow to allow a direct comparison of the low fluorescence intensity associated with the vertically aligned regions with the high intensity produced by the horizontally aligned regions. At lower rates of flow, the intensity measured shows a double peaked curve against the displacement of the focal plane from the instrument's start position, as in Fig. 6. The two peaks indicate slightly tilted regions, while the dark midplane signal indicates a vertically aligned region. In stark contrast, the intensity is maximum at the midplane when measured at the higher rates of flow, indicating a horizontally aligned region in accordance with the predicted $H$ state.

\section{FCPM flow velocity measurements}

In order to quantify the fluid velocity distribution across the cell depth the FCPM measurements were taken a step further by distributing a low $(0.5 \%)$ concentration of FITC coated $1 \mu \mathrm{m}$ diameter beads (Sigma-Aldrich) in a dyedoped sample of ZLI-2806 (Merck) liquid crystal. These beads were specifically chosen due to their miscibility with the liquid crystal and their accessible excitation (490 nm) and emission $(525 \mathrm{~nm})$ wavelengths. Once filled via the syringe-drive system the cell was left for one hour to form a uniformly aligned nonflowing homeotropic state. The cell was oriented with the polarization direction of the excitation beam along the $x$ axis and parallel to the direction of flow and the syringe set to a volumetric flow rate of $2 \mu$ l per hour and the entire system was left for $30 \mathrm{~min}$.

The flow velocity was determined by focusing the FCPM system at a given depth into the cell to produce an image of a $160 \mu \mathrm{m} \times 160 \mu \mathrm{m}$ region of the cell in the $x-y$ plane far from the walls with a resolution of $512 \times 512$ pixels. The system was set to scan the region continuously, resulting in a time interval of $0.75 \mathrm{~s}$ between each sequential image of a point on the plane. Fluorescence intensity data was collected in this way over $\sim 40 \mathrm{~s}$ with the transmitted image of the plane of the cell collected simultaneously using a broad-band PMT. The system was then refocused to a new position along the $z$ axis and this was repeated until data from across the whole cell was collected. The volumetric flow rate of the syringe drive was then changed to the next value required, the system was left for $30 \mathrm{~min}$, and the whole process was repeated.

To determine the velocity of the beads, and hence the associated fluid velocity, for each set of $x-y$ plane images at a given flow rate and depth, the $x-y$ coordinate of a single bead in focus was determined using image processing software (ImageJ). The position of the bead in the next image was then measured and this continued until either the marker had traveled out of the field of view or the data collection had ceased. As expected, it was found that the beads moved in a clear linear path parallel to the walls of the channel. The average number of pixels moved per frame and the standard deviation were calculated and then converted into meters per

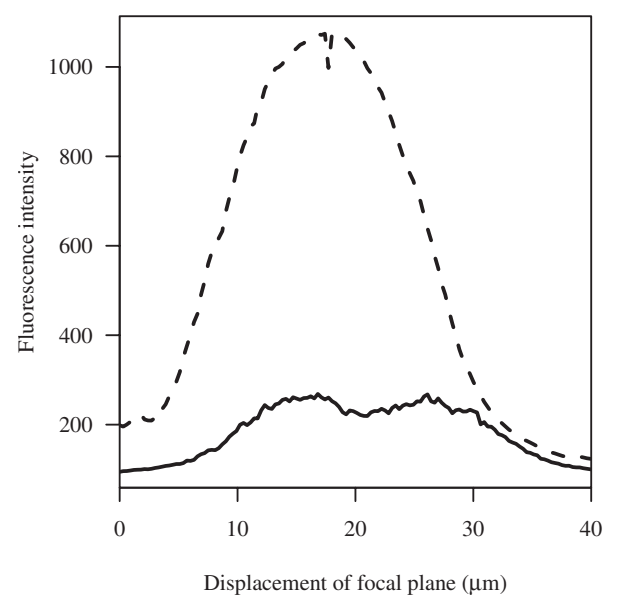

FIG. 6. FCPM data. The low amplitude double peaked curve plots measurements taken below the critical flow rate at $6 \mu \mathrm{l} / \mathrm{hr}$ and indicates a $V$ state. Just above the critical flow rate, at $8 \mu \mathrm{l} / \mathrm{hr}$, the cell fluoresces strongly near the midplane (dashed curve), implying that the liquid crystal is horizontally aligned there. 


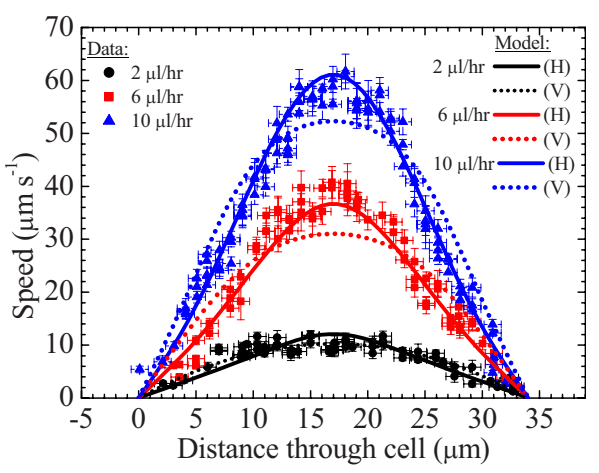

FIG. 7. (Color online) Measured (symbol) and modeled (solid line) flow velocity profile as a function of depth for a $160 \mu \mathrm{m}$ $\times 160 \mu \mathrm{m}$ region of a homeotropically aligned liquid crystal cell for selected pressure-driven volumetric flow rates. The legend shows either $V$-state or $H$-state alignment used for the modeling. Each separate data point is for an individual bead.

second. This was repeated with several other beads to give a spread of measured velocities for that depth.

The resulting speed measured as a function of depth for volumetric flow rates of $2 \mu \mathrm{l} / \mathrm{hr}, 6 \mu \mathrm{l} / \mathrm{hr}$, and $10 \mu \mathrm{l} / \mathrm{hr}$ are shown in Fig. 7. Each profile is highly symmetrical about the midpoint of the cell and corresponds to a cell thickness of around $34 \mu \mathrm{m}$. Due to the complex interaction between the director and the resulting shear forces governing the fluid flow, the distribution of velocities across the cell for the two types of alignment are noticeably different. This has been highlighted by modeling the Poiseuille flow through this homeotropically aligned cell using Ericksen-Leslie theory described previously. As the specific viscoelastic parameters for ZLI-2806 were not available, the parameters for 5CB (given in Table I) were used as a good approximation. The solid and dashed lines in Fig. 7 show how the $H$ - and $V$-state flow velocity profiles for volumetric flow rates of $2 \mu \mathrm{l} / \mathrm{hr}$, $6 \mu \mathrm{l} / \mathrm{hr}$, and $10 \mu \mathrm{l} / \mathrm{hr}$ vary. For the $V$-state flow, the velocity distribution has a smoothly rounded profile whereas the $H$-state flow is distinctly more peaked in shape. For the two higher volumetric flow rates the $H$-state profile shows excellent agreement with the measured velocity profiles. For the lower flow rate, the difference between the two profiles is less distinct but the flatter profile attributed to the $V$-state flow shows the best agreement with the measured flow speeds.

\section{CONCLUSIONS}

In summary, a transition from a near-vertical $(V)$ state to a partly horizontal $(H)$ state can be induced in a homeotropically aligned nematic liquid crystal undergoing Poiseuille flow through a microchannel by driving it above a critical flow rate. These states are mirror symmetric about the cell's midplane, but otherwise quite different, and were therefore readily identified by using conoscopy and fluorescence confocal polarizing microscopy. Time lapse imaging allowed the first experimental observation of the velocity distribution across the channel for each configuration. The results agreed well with theoretical data produced by solving the EricksenLeslie equations numerically for a simplified, onedimensional model, and the rate of flow at which the transition would occur was predicted and verified.

\section{ACKNOWLEDGMENTS}

The authors would like to acknowledge funding from the EPSRC. S.L.C. would also like to thank HP Labs, Bristol for funding.
[1] S. A. Jewell and J. R. Sambles, Appl. Phys. Lett. 84, 46 (2004).

[2] S. A. Jewell and J. R. Sambles, Mol. Cryst. Liq. Cryst. 477, 57 (2007).

[3] O. P. Pishnyak, S. Tang, J. R. Kelly, S. V. Shiyanovskii, and O. D. Lavrentovich, Phys. Rev. Lett. 99, 127802 (2007).

[4] I. Dierking et al., Phys. Rev. E 76, 021707 (2007).

[5] J. Feng and L. G. Leal, Phys. Fluids 11, 2821 (1999).

[6] J. Fukuda et al., J. Phys.: Condens. Matter 16, S1957 (2004).

[7] D. Lasne, A. Maali, Y. Amarouchene, L. Cognet, B. Lounis, and H. Kellay, Phys. Rev. Lett. 100, 214502 (2008).
[8] F. M. Leslie, Arch. Ration. Mech. Anal. 28, 265 (1968).

[9] I. W. Stewart, The Static and Dynamic Continuum Theory of Liquid Crystals (Taylor and Francis, London, 2004).

[10] C. Z. van Doorn, J. Appl. Phys. 46, 3738 (1975).

[11] C. Denniston, E. Orlandini, and J. M. Yeomans, Comput. Theor. Polym. Sci. 11, 389 (2001).

[12] G. Waton et al., Mol. Cryst. Liq. Cryst. 78, 237 (1981).

[13] I. I. Smalyukh, S. V. Shiyanovskii, and O. D. Lavrentovich, Chem. Phys. Lett. 336, 88 (2001).

[14] S. A. Jewell and J. R. Sambles, Phys. Rev. E 78, 012701 (2008). 